\title{
Richtig für die Gesundheit
}

\section{vorsorgen}

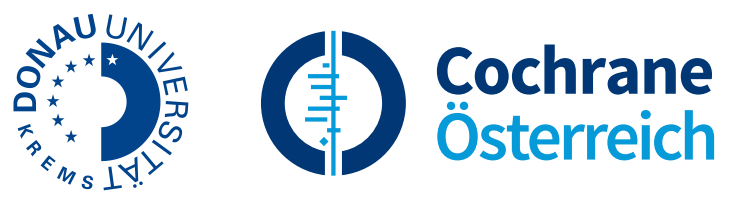

\section{H}

inter einer Vorsorgeuntersuchung steht das Interesse, Erkrankungen früh zu entdecken und die Risikofaktoren dafür zu beurteilen. Doch nicht jede Vorsorgeuntersuchung ist immer sinnvoll, in manchen Fällen kann sie sogar schaden.

Ein Aspekt ist besonders wichtig, um einzuschätzen, ob eine Untersuchung notwendig ist: Nur bei Krankheiten, die so früh wie möglich behandelt werden müssen, bringt eine Früherkennungsuntersuchung Vorteile. Denn auch Untersuchungen können schaden und ihre Ergebnisse sind nicht immer zuverlässig. Es kann zu falsch-positiven Ergebnissen kommen und ein Mensch wird als krank eingestuft, obwohl er es nicht ist. Überdiagnosen können bewirken, dass Krankheiten behandelt werden, obwohl sie nie zu Beschwerden geführt hätten. All das sollte in der Praxis berücksichtigt werden, bevor eine Vorsorgeuntersuchung angeordnet wird.

\section{Gemeinsam gut entscheiden in der Vorsorgemedizin}

Die Initiative „Gemeinsam gut entscheiden" und die Österreichische Gesellschaft für Public Health weisen auf sieben Vorsorgeuntersuchungen hin, für die vielfach kein Nutzen nachweisbar ist:

\section{Bakterien im Harn}

Bei älteren Menschen ab 65 Jahren finden sich relativ häufig Bakterien im Harn. Sie haben dabei aber keine Beschwerden eines Harnwegsinfekts. Eine Behandlung mit Antibiotika ist in diesem Fall nicht notwendig. Auch eine Untersuchung ohne vorhandene Symptome macht keinen Sinn.

\section{Osteoporose-Test unter $\mathbf{5 0}$ Jahren}

Bei gesunden Menschen unter 50 Jahren kommt Osteoporose selten vor. Es ist daher nicht sinnvoll, in diesem Alter ohne besonderes Risiko nach verminderter Knochendichte zu suchen.

\section{Vitamin-D-Mangel}

Bei Menschen ohne Beschwerden muss der Vitamin-D-Spiegel im Blut nicht überprüft werden. Für eine ausreichende Versorgung genügen zumeist Sonne und abwechslungsreiche Ernährung.

\section{Früherkennung von Typ-2-Diabetes}

Nur bei erhöhtem Risiko für Typ-2-Diabetes empfiehlt sich eine Früherkennungsuntersuchung. Das trifft zum Beispiel auf Menschen zu, deren Body-Mass-Index bei $25 \mathrm{~kg} / \mathrm{m}^{2}$ oder darüber liegt, oder wenn nahe Verwandte von einem Typ-2-Diabetes betroffen sind.

\section{Gamma-GT zur Ermittlung von gesundheitsgefährdendem Alkoholkonsum}

Ist die Leber geschädigt, ist die GammaGT im Blut erhöht. Wird dieser Wert in der allgemeinen Bevölkerung untersucht, haben viele einen erhöhten Wert, obwohl sie keinen oder wenig Alkohol trinken. Gamma-GT eignet sich nicht dazu, um Menschen zu identifizieren, die zu viel Alkohol trinken.

\section{Früherkennung chronischer Nierenerkrankung}

Vorsorglich die Nierenfunktion zu überprüfen empfiehlt sich nur für Menschen, die ein erhöhtes Risiko für eine Erkrankung der Nieren haben.

\section{Vorsorge und Früherkennung} bei einer Depression

Hat ein Mensch keine oder kaum Beschwerden, sollte er nicht auf eine Depression hin untersucht werden, da falschpositive Diagnosen häufig sind. Studien zeigen auch, dass eine frühzeitige Diagnose keine spürbaren Vorteile für die Betroffenen hat.

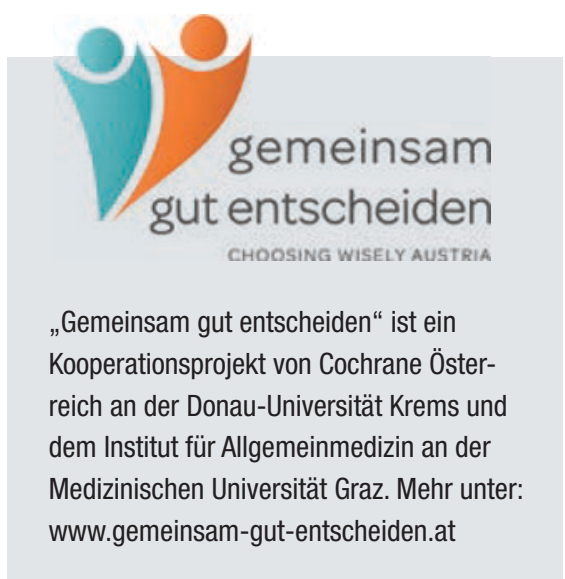

Autorin:

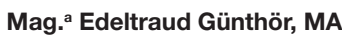

Projektmitarbeiterin bei

"Gemeinsam gut entscheiden“

Department für Evidenzbasierte Medizin und Evaluation

Donau-Universität Krems

edeltraud.guenthoer@donau-uni.ac.at 